\title{
E. SAN JUAN, JR. AT ANG DISKURSONG KULTURANG POPULAR
}

\author{
Rolando B. Tolentino \\ University of the Philippines Film Institute \\ roland.tolentino@gmail.com
}

\begin{abstract}
While E. San Juan, Jr. admits that he has done little scholarship on popular culture, my contention is that his body of works-especially in the areas of cultural revolution, Third World literature, and diaspora-provides a substantial contribution and intervention in Philippine cultural studies and popular culture studies. His writings on these typify the critical resolve in the cultural turns as experienced in the rise of cultural studies. In particular, it is useful to the analysis of popular culture, representing a framework that has integrity, even in the mapping of the contemporary popularity of social media platforms. This personal essay analyzes the various stages in the rise and development of cultural studies, including the mediations of San Juan in his related critiques, including the place and placement of cultural revolution. The field of popular culture studies owes San Juan a huge gratitude with his early appreciation and understanding that culture is political, and that the political's call is for national social transformation.
\end{abstract}

\section{Keywords}

araling kultural, E. San Juan, Jr., kulturang popular, pambansang demokrasya

\begin{abstract}
About the Author
Rolando B. Tolentino is faculty of the University of the Philippines Film Institute and former dean of the UP College of Mass Communication. He has taught at Osaka University and National University of Singapore. His research interests include Philippine literature, popular culture, cinema and media, interfacing national and transnational issues. He writes fiction and creative non-fiction, and is a fellow of the UP Institute of Creative Writing. He is a member of the Manunuri ng Pelikulang Pilipino (Filipino Film Critics Group), Congress of Teachers and Educators for Nationalism and Democracy (CONTEND-UP), and Altermidya (People's Alternative Media Network).
\end{abstract}


Noong 1990 s na nag-aaral ako sa U.S. para sa doktorado, may kakaibang reputasyon na si E. San Juan, Jr. Hindi ko siya nakasabayan nang sa una kong taon ay sa Bowling Green State University ako napadpad. Minsan lang kami nagtagpo roon, nang magka-forum sa unibersidad at isa siya sa mga tagapagsalita. Ewan ko kung naalaala pa niyang naging guro ko siya sa una kong pagtatangka sa masteral sa U.P., at kinailangang mag-withdraw dahil ang daming nangyayari sa bansa noon, at ako ay full-time na aktibista. Isa rin siya sa mga haunting figures sa aking pagsusulat. Natandaan ko pa nang magpasa ako ng artikulo sa Amerasia Journal, ang sinabi ng Filipino American na namamahala sa publikasyon ay "parang San Juan" ang pagkasulat ko. At heto ang pagkakataong mag-full circle ang multo ni San Juan sa akin, ang pangagailangang tasahin ko ang kanyang napakahalagang kontribusyon sa diskursong kulturang popular.

Sa katunayan, at aminado naman si San Juan, na wala siyang mga artikulo hinggil sa kulturang popular. Ang aking kontensyon, magpakaganito man, ay nananatili ang kanyang presensya sa pagkahubog at pagkalatag ng kritika sa diskursong kulturang popular. Kultural ang pangunahing larangan ng kontribusyon sa kritika ng bansa ni San Juan, at ito ang hindi matatawarang substansya ng kanyang kritikal na pamanana ilagay ang usaping kultural sa usaping politikal sa pambansang kalagayan o ang usapin ng kritika sa kasaysayan at pagkaunlad ng kultura sa pangangailangan at tunguhin para sa pambansang pagbabago.

Ang kanyang kritika ng rebolusyong kultural, panitikan ng Ikatlong Daigdig, formasyon at transformasyon ng bansa, diaspora, at iba pa ay may kakayahang makapaglatag ng kritisismo sa politika na kultural (cultural politics) na siya ring mayabong na arena ng kritisismo sa kulturang popular. Na kahit pa nagkaroon ng malalaking paradigmatikong pagbaybay sa kulturang popular via araling kultural (cultural studies) - postmodern shift, negosiasyon at making do, poststructuralist postmodernism, postmodern cultural turn, at kritika ng metastruktura-ay masasabing napangunahan na ni San Juan ang mga pagbabago sa iba't ibang yugto ng araling kultural tungo sa inaakalang kasalukuyang tunguhin nito sa kritika ng kapitalismo. Sa pagtalakay sa ibaba, matutunghayan na ang pinaunlad na paninindigan ni San Juan sa kanyang kritika ng Third Word literature, diaspora, at maging ang politikal na kondisyon ng posibilidad sa mga usaping kultura at kultural sa bansa-gamit ang sentral na kritikal na imahen ng rebolusyong kultural-ay ang kasalukuyang lunan ng interbensyon sa pagsusuri at pag-aaral ng kulturang popular: na ito ay sityo ng kontestasyon ng mga uri, at kung gayon ay hindi pantay at makatarungan ang panlipunan at historikal na interes at relasyon dito, at nangangailangan ng paglalatag ng etika ng pagbabago at transformasyon. Kung gayon, ang katapatan sa batayang Marxismo at kaisipang Mao Zedong na inilatag ni San Juan para sa politika na kultural ang siya pa ring saligang prinsipyo na giya sa muling pagtatagpo sa diskursong kulturang popular. 
Sa sanaysay na ito, una kong tatahakin ang personal na karanasan sa araling kultural at ang impakt nito sa araling kulturang popular. Dito maaninag ang remisyon ng araling kultural sa pangkalahatan, at maging sa mga yugto na pagkaunlad nito sa bansa at sa global na antas. Ilalatag ko ang lugar ng kritika ni San Juan sa kulturang popular sa pamamagitan ng paglalatag ng kanyang batayang idea at konseptwalisasyon ng rebolusyong kultural na nilikha niya bilang saligan ng kanyang kritika sa panitikan ng Ikatlong Daigdig, formasyon at transformasyon ng bansa, diaspora, at iba pa. Ang pananatiling katalasan ng kritika ni San Juan ang siya ring batong papatalas sa talim ng araling kulturang popular na ang diin ay ang kritika ng kapitalismo sa dating (affect) nito sa formasyon at transformasyon ng identidad, bansa, at globalidad.

\section{ANG SERIALISASYON NG ARALING KULTURAL}

Nang ako ay nagmamasteral sa De La Salle University sa programa sa araling Pilipinas, natunghayan ko sa unang pagkakataon ang kritisismong pampanitikan. At ang dulo o hangganan nito noon ay ang postmodernismo-na ang referensiya ay shopping o pamimili ng mga item na maaring gamitin ng kritiko para makapaglahad ng kanyang kritika sa bagay na pinag-aaralan, ang texto. Dulo ng 1980 os ito, at naalaala ko rin ang isang pagkakataon na may sharing session na isinagawa ang dalawa o tatlong gurong kritiko ng unibersidad na galing sa cultural exchange sa United Kingdom, at kung saan ay napag-alaman nila ang araling kultural. Tadtad ang naging sesyon sa isang klasrum ng bagong konsepto, kritiko, at daluyan ng kritika: anti-metastructures (basta lahat ng nakacaps, tulad ng Panitikan, Patriarka, Kapitalismo, Sosyalismo, Kanluraning Kritika, at iba pa), British cultural studies, Birmingham school, ang ipinagkaiba nito sa French cultural studies at American deconstructionism, E.P. Thompson, Raymond Williams, Stuart Hall, at iba pa bilang ilan sa naaalala ko. Mataas ang energy level ng sharing, dahil parang gusto naming tagapakinig at relihiyosong kumukuha ng notes na lumusob sa library kahit pa wala pang libro sa ipinapakilalang paksa rito. $\mathrm{O}$ sila pa lamang nangunguna sa sharing ang may hawak ng libro, at handa naman ipa-xerox ang kabuuan nito sa aming tagapakinig.

Magkakaroon ng kultural na halaga ang araling kultural dahil sa bago ito, at may pangako kundi man posibilidad na may makabagong tinutugunan na pangangailangan laban sa mga nakagawiang ortodoxiya: marxismo sa pangunahin, at ang pagiging pantay ng iba pang kategorya, tulad ng feminismo, at sa maikling panahon, ang pamamayagpag ng araling bakla, o sa mas deterministikong papel ng sexualidad at kasarian, lahi at etnisidad, at ang pagbabalik ng kategorya ng subkultura, lalo na ang kabataang subkultura. Tinanggal ang pribilehiyo ng uri, at 
ipinantay sa iba pang kultural na kategorya, o mas binigyan-pribilehiyo ang iba pang kultural na kategorya dahil nga wala dapat na kinikilalang metastruktura. At ang naging 'biktima' nitong anti-metastruktura sa kritika ay ang marxismo at ang naging preferensiya ng mga kritiko simula sa kilusang Filipinisasyon noong 1960 s na nanghihimok na bigyan ng angkop na prioritisasyon ang mga paksain at temang Filipino, lalo na ang wikang Filipino, sa produksyon ng kontra-kanluranin na kaalaman sa mga disiplina.

Naging bahagi ako ng kritikal na sandali na naghuhudyat ng paradigmatikong paling sa kritika ng panitikan, kultura, at kulturang popular tungo sa mikroidentidad at politikang panlipunan. May pagtataka ako kung pwede na nga bang isa na lamang sa mga kultural na kategorya ang usaping uri, kapantay lalo na ng uri at kasarian na aalagwa sa mga panahong ito. Ang praktikal na karanasan ko sa aktibismo ang magsasabi na pangunahin na usapin at balangkas pa rin sa kritika ay ang uri, tulad ng inihudyat ng kritisismo at kritikal na praxis ni San Juan. Sa isang banda, ang mga magiging malinaw na makalumang kritiko-ang mga nakatali pa rin sa formalistikong gawi-ay may bulgar at pedestrianong turing sa usaping kultura, kulturang popular, at mga kultural na kategorya. Tulad ng turing nila sa hindi humanistiko o syentifiko ang kritika ng marxista, gayundin ang turing sa araling kultural bilang kritikal na balangkas, lalo pa kung ang sinusuri ay mga bulgar at pedestrianong paksa at penomenon sa kulturang popular! Tanging sa Departamento ng Filipino at Panitikan ng Pilipinas sa Kolehiyo ng Arte at Literatura, at sa Departamento ng Sosyolohiya sa Kolehiyo ng Agham Panlipunan at Pilosopiya ng U.P. Diliman mayroong kurso sa kulturang popular. Ang araling kultural ang lalabas na kritikal na balangkas sa mga skolarsyip, lalo na libro, na lumabas sa kulturang popular.

May mga guro ako na pinaghahalawan ng tradisyon sa malikhaing pagsulat at kritika at simpleng usapin ito ng pagkapukaw ng aking interes para pumihit sa kanilang kahalintulad na gawain, at bukal na pagyayamanin ito. May itinuturing din akong guro kahit hindi ko sila naging guro sa klase na nagawa rin ang pagkapunla ng interes sa mga paksa, pagpapalawig, at pagturo ng direksyon ng gawain para sa hinaharap. Ito ang mga multo at guni-guni para sa akin-multo dahil hindi ko naabutan ang mga historikal na figura (Jose Rizal, Andres Bonifacio, Amado Hernandez, Jose Maria Sison); at multo dahil buhay pa sila pero may "pagmumulto" (pagpaparamdam at maglalagi) sa akin, tulad ni Edel Garcellano, Monico Atienza, at San Juan. Hindi ko sila naging guro o maituturing na malapit na kaibigan, may senioridad sila sa akin na isang katangian ng mga henerasyon ng kritiko sa bansa, pero ang kanilang akda at maging ang mismong presensya sa mga publikong presentasyon, lalo na sa malayang talakayan, ay nagsilbi na inspirasyon at aspirasyon sa aking tinahak at tatahakin pa sa kritika at pagiging mamamayan sa bansang ito. Marxista ang aking mga hinahangaang multo dahil may nagtagpo sa 
sangandaan ng kritikal na sandali ang kanila at aking kritika. Mayaman ang ambag ng marxistang kritiko at panunuri sa kritika ng bansa, at patuloy ang kanilang kritikal na interbensyon na ang mga batayang usapin ng makauring panunuri at kahilingan na pambansang demokrasya at pagbabago ay nananatiling makabuluhan sa kasalukuyan at sa hinaharap.

Sa dekada 1970 ay ito ang naging direksyon ng kritika sa panitikan, humanidades, sikolohiya, pilosopiya, syensyang panlipunan, at sa mas limitadong kapasidad, sa syensya mismo. Kung sisipatin, may katapatan ang pagkaunlad ng kritika sa pagkaunlad ng mga kilusang panlipunan sa bansa, at ang marxismong pangunahing gamit para aralin ang panitikan, mga disiplina, at ang lipunan at kasaysayan. Ang kaganapang politika ang siyang impetus ng pagkahubog at substansasyon sa kritika. Ito ang sentral na ekolohiya at kapaligiran kung saan yumabong ang mga disiplina na may pangunahing fokus sa artikulasyon at elaborasyon ng pagka-Filipino sa mga ito.

Bahagi si San Juan sa intelektwal na sandali at kritikal na momentum na ito. Matapos niyang makuha ang kanyang masterado at doktorado sa Harvard University ay nagturo ito sa iba't ibang unibersidad sa U.S. Kahit hindi bumalik si San Juan sa Pilipinas ay bumabalik at pabalikbalik ito. Kaya patuloy sa kanyang intelektwal na pagkaunlad ang piniling afinidad na bumabalik at pabalikbalik sa bansa, at sa historikal at panlipunang pinagdadaanan nito. Maging ang kritika ni San Juan ay matibay ang pagkakawing sa historikal at panlipunang problematisasyon ng panitikan at kultura sa mas malawak at malalim na ugnay sa makauring problema at pagsusuri ng mismong kasaysayan ng at lipunan sa bansa.

Kinakatawan ni San Juan ang tradisyon ng kritiko, manunulat at intelektwal na aktibista at rebolusyonaryo gaya nang mga nauna sa kanya. Sa kasaysayan ng kritika, formasyon at transformasyon ng intelekwal, may mahalagang daluyan na ang mga intelektwal ay reformista, rebolusyonaryo, organisador, unyonista, sosyalista, komunista, o aktibista. Hinog ang mga kritikal na kondisyon noon pa mang nagsisimula ang pambansang demokratikong kilusan noong 1960s, ang pagkaunlad ng aktibismo sa U.P., ang deklarasyon ng batas militar noong 1972, ang pagpapaigting ng diktaduryang Marcos sa 1970s, pagkasadsad nito noong 1986, ang pagkahati ng kilusang Kaliwa sa dulo ng 1980s, ang institusyonalisasyon ng neoliberalismo sa panahong ito at higit na pagpapalawak ng operasyon nito sa susunod na mga dekada, ang kilusang rektifikasyon noong 1990s, ang higit na pagkasadlak ng mamamayan sa pandudusta ng hinalal nitong mga pambansang pamunuan, ang paratihang presensya ng U.S. sa kalakarang politika, ekonomiya at kultura, ang pumapaimbabaw na presensya ng China, at iba pa. Pinili ni San Juan maging bahagi nitong mga politikal na transformasyon ng bansa at pambansang demokratikong kilusan noon at hanggang sa kasalukuyan, at ang politikal na 
intelektwal na paninindigang ito ang siyang nagbibigay na patuloy na halaga at sikhay sa kritika ni San Juan.

Huling yugto ng dekada 1980 naipakilala ang postmodernismo at ang paling sa araling kultural, na siyang mamamayagpag bilang balangkas ng pagsusuri sa kritika sa panitikan at iba pang disiplina. Ang Kaliwa ay nahati sa dalawang faksyon: ang rejectionista na ayaw ng sentralismo ng Partido Komunista ng Pilipinas, pumailanlang sa rebisyonismo ng pangunahing paglahok sa eleksyon at pambansang pamamahala; at ang reafirmista na bumalik sa mga batayang pagaaral para muling makapag-organisa sa hayag at di-hayag na antas. Mamamayagpag ang reafirmista bilang politikal na intelektwal na daluyan ng kritisismo sa dekada 200o. At may kahalintulad na paradigmatikong pagpaling din sa araling kultural na namayagpag sa panahong ito. Una, ang introduksyon ng araling kultural sa yugto ng postmodernismo na kritika ay tinangka ng mga kritiko na lumikha ng panibagong balangkas ng pag-aaral, at sa isang unibersidad, ng isang programa para sa undergraduate at graduate. Sa pagtatangkang ito, tinalikdan ang mga metastruktura maging sa kritisismo, tulad ng formalismo, bagong kritisismo, at marxismo, para bigyan-pribilehiyo ang admixture o halo-halo (pragmatikong diin na kung ano ang may kabuluhan sa pinag-aaralang texto) na panunuri. Itinuring pa rin na ang pinag-aaralan ay texto, at ang diin ay textualidad o pagkabuo ng diskurso sa loob ng texto, at intertextual o pagkaugnay sa iba pang texto.

Ang sumunod na yugto sa paradigmatikong paling ng araling kultural sa bansa, sa aking palagay, ay ang pag-alagwa nito na hiwalay sa postmodernismo na kritika. Naging autonomus na kritika ang araling kultural, nawala na sa purview ng postmodernismo. Ang naging arena ng paksa ay kulturang popular dahil sa postmodernismong simulain nito, at ang mga penomenong kultural na itinuturing na sinasambulat nitong postmodernong turn sa kultura, tulad ng malling; mga makabagong karanasan, palabas sa telebisyon, gadget na tinatangkilikng pangunahin ay kabataan; postmodernismong panitikan na tumatalakay sa ganito; at iba pa. Ang diin ng araling kultural ay negosiasyon o ang pagtahak ng liminal na erya na ang itaas o nasa kapangyarihan ay nadidispalto ang kanilang kapangyarihan at ng nasa ibaba na nakakapagpatotoo ng kontraryong kapangyarihan. Tinawag din itong "making do" (Michel de Certeau) at subersyon (John Fiske) gayong sa pangunahin, ang impetus ng negosiasyon ay kay Antonio Gramsci, at parang may panahon pa na ang anumang kontraryong bagay na ginagawa o pinipihit ng mga nasa ibaba ay tinaguriang radikal na reformulasyon ng relasyong pangkapangyarihan.

Ang ikatlong yugto na may paradigmatikong paling ay nauukol sa poststukturalistang pagpipihit sa araling kultural. Rito ay tinatanaw ang lahat ng pagsusuri na pantay, na ang kontensyon ni kritika A ay kapantay ng pagpapahalaga sa kontensyon ni kritika B, K, D, at iba pa sa parehong textong pinag-aaralan. Halaw 
kay Michel Foucault sa liberal na aplikasyon dito ng isinaad na ang "power is nowhere and everywhere," na ang pagkaunawa sa kritika ay walang iisang humahawak ng poder ng kapangyarihan. At bilang kontraryong kapangyarihan, ang lahat ay dapat may akses sa formulasyon ng kanilang pagkaunawa sa diskurso ng kapangyarihan. Ang isinasaad din nito ay ang pag-alingawngaw ng unang yugto ng araling kultural: bilang anti-metanaratibo at anti-metastruktura ng iisang gahum sa kritika, na sa pangunahin ay nagpapakahulugan bilang anti-marxismo. Ang resulta ng pagpapantay sa balangkas ng pagsusuri ay ang pagkabalaho ng mismong kritikal na diin nito sa araling kultural, ibig sabihin, nawala ang usapin ng etika o prioritisasyon ng halaga at kabuluhan ng balangkas na gamit sa sinusuring texto: na ang queer reading kay Manny Pacquiao, halimbawa, ay pantay din sa makauri, anti-feminista, liberal na araling etniko at panlahi, pagsadlak ng araling pamamahala, at iba pa dahil nga ang bawat isa ay may kapantayang halaga at kabuluhan sa paglalahad ng kani-kanilang diskurso at interpretasyon ng diskursong kapangyarihan.

Ito ang nagdulot ng cultural turn o tuluyan nang pagkasadlak ng araling kultural na nawalan na ito ng talas para balangkasin, idikonstrakt, himayin, at i-reorder ang mga bagaybagay at kaantasan ng kapangyarihan at mismong diskurso. Ito ang sistema ng pagpapantay (equivalence) sa araling kultural, at ang propensidad na muling gawing textual ang pagsusuri. Ang naidulot ng ikatlong yugto ay ang pagpasok ng field work at etnograpiya sa paghahanap ng pang-araw-araw na modalidad ng subersyon. Sa ikaapat na yugto, ipinasok pa rin ito pero wala nang moral na paghuhusga, o tulad sa antropolohiya, nauwi ang pagsusuri sa paglalahad lamang ng mga naratibo ng pagtutugma o kontestasyon hinggil sa pinag-aaralang penonemon o thick description. Nakatunghay na ako ng pag-aaral hinggil sa sex scandal videos sa bansa, halimbawa, na ang ginawa ng antropologo ay lumikha lamang ng mga kategorya para sa pagdanas ng iba't ibang klaseng sex scandal videos. Walang pagsusuri ng politika ng kultural sa penomenong pinag-aaralan, o kung ano ang spekulasyon ng antropologo kung bakit ganito ang pagkamundo ng mga kategoryang kanyang pinag-aaralan. Nawala ang mas malaking usapin ng Kapangyarihan o ang orkestrasyon ng aktwal na aparato ng politika, ekonomiya at kultura sa bansa at mundo.

Walang moral na paghuhusga sa hegemoniya dahil ang tinatalakay ay ang epekto ng hegemoniya sa nasa ibaba. Kontraryo ito sa politikal na pagsusuri sa tradisyon ng kasaysayan ng intelektwal sa bansa, ang pakikilahok ng intelektwal sa panlipunan at historikal na pagbabago, at ang propensidad ng mayoryang akda sa tradisyong ito na maging hayagang politikal at polemical sa "our urgent task." Nagkaroon muli ng valuasyon sa metastruktura at metanaratibo-sa partikular ng neoliberal na globalisasyon, late capitalism, kritika ng kapitalismo, neokolonialismo at imperialism, at maging reintroduksyon ng makabagong marxismong may hawig tungo sa pilosopiya ng sosyalismo at komunismo (Slavoj Zizek at Allan Badiou, 
halimbawa) - na nakapagbigay-talas muli sa araling kultural at sa dati nitong kapasidad sa politikal na kritisismo na halaw sa kanyang kritikal na tradisyon. Naging sentral na usapin pa rin ang kulturang popular lalo pa sa panahon na sa 2010, higit na umalagwa ang fasinasyon, seduksyon, at pamamayagpag ng Internet, social media platforms, at smart phones sa pang-araw-araw na buhay ng kabataan at mamamayan. Ito ang kabalintunaan ng reintroduksyon ng marxismo bilang teoretikal na giya sa araling kultural: sa edad na akala ng hegemoniya ay nalusaw na ang kolektibo, kasaysayan at politikal, saka pa nag-comeback ang marxismo para sa mas malawakang artikulasyon ng pagkahumaling sa kapital sa pangaraw-araw, at ang paglikha sa komunismo at "another world is possible" bilang kontraryong pagmumundo sa kasalukuyang pagkasadlak. Ang aral na matutunan kay San Juan na hindi natinag sa pagiging fasyonista sa araling kultural ay ang pag-angkla ng kritika sa politikal na may dalawang aspekto: una, ang pagsusuri sa mismong politika ng penomenon, at ang representasyon nito sa akdang pinagaaralan o kung paano ito naging representatibo ng isang mahalagang sangay ng pagsasakapangyarihan sa panitikan at pamahalaan, ito ang usaping kontraryo o paglalahad ng kontra-hegemoniya na pagsusuri; at ikalawa, dahil ang politika ay kontradiktoryo, may pagkiling sa kapangyarihan, may kahinaan o kamalian sa katarungang panlipunan, kailangan itong palitan, at inaakda rin ang alternatibo o ang paglalahad ng alternatibong posibilidad lampas sa kasalukuyang pagkasadlak, at kung gayon, ang aplikasyon sa mas makatarungang hinaharap.

Ang presensya ni San Juan sa kritika ng bansa ay konkreto dahil ang bansa ang kanyang pinaghahalawan ng kanyang kritika. Walang paksa at balangkas ang hindi nakaugnay para sa kanyang kritikal na praxis. Ang pisikal na presensya niya-ang paratihang nagbabalik na skolar na may publiko at kabahagi ng kilusang mamamayan-ang siyang tumimo rin sa akin na maari palang pagsabayin, kundi man hindi dapat magkahiwalay, ang paninindigan sa kritika at pagiging mamamayan sa paninindigan para sa bayan. Ako man ay naghahanap ng kritikal na lente sa kritika at pagiging mamamayan, at si San Juan ang isa sa makabuluhang panandangbato na siyang nagiya sa akin para matunghayan na ang hinahanap ko ay nasa tabi ko lang, kundi man nasa loob ko na rin pala.

\section{ANG NAWAWALA, NATAGPUAN}

Epektibong natibag ang metastruktura na nagma-matter o nakakapagbigayhalaga sa tao, bagay, lunan, at pangyayari. Ito ang mga aparato ng politika, ekonomiya at kultura, at sa ganitong usapin din binabaybay ni San Juan ang artikulasyon ng kontraryong aparato-isasyon para makapaglahad ng alternatibong posibilidad sa hindi makatarungang kasalukuyan. Ito ang nawala sa araling kultural, o winala ng 
kontra-Kaliwa na kritiko na namayagpag din kahit sa aktwal na pagsibol at pagunlad ng postmoderniso at araling kultural. Ang nawawala hindi naman kusang nawala (pangnagdaan) at nawawala (pangkasalukuyan). May motibasyon kung bakit ito nawala at nawawala, at ito ang depolitisasyon ng kritika at kilusang panlipunan (kasama ang civil society) sa ngalan ng mabuting pamamahala na may inaasahang resultang mabuting pagkamamamayan. Kung depolitisado ang kritika at kilusang panlipunan, walang pangangailangan ng politikal at kultural na transformasyon, at kung gayon, may pagtanggap sa operasyonalisasyon ng hegemoniya. Ang ilan sa mga kritiko at skolarsyip na nalikha ng mga ito ay yaong nagdidiin sa pagmumundo ng penomenong pinag-aaralan bilang unibersal, $o$ bagong unibersal na paghahalawan ng kahalintulad na pag-aaral. Kumbaga sa humanismong formalismo at syentifismo ng bagong kritisismo, ang mga kalakaran maging sa araling kultural ay ang pagsusuri ay nakapaloob lamang sa organikong kaisahan ng mga elementong bumubuo ng inilalatag na Isa at Kaiba (self, other). Epektibong naiwalay ang historikal at panlipunang pwersa sa labas ng kaisahang ito, ang tanging politikal na itinira ay ang politika ng pagsasantabi kundi man biktimisasyon sa loob lamang ng penomenon, walang politika sa labas nito. Napapagtagumpayan ang depolitisasyon ng kritika at kilusang panlipunan kapag tinanggal ang napakahalaga at napakamakabuluhang usapin ng uri, at makauring pagsusuri. Nawawala ang talas ng kritika dahil uri ang nagbibigay-talas para ang sinusuri ay magkaroon ng potensyal sa usapin ng transformasyong historikal at panlipunan.

Sa isang sanaysay ni San Juan, natumbok niya ang pagkawala ng ngipin ng araling kultural dahil ito ang hindi niya tinalikdan sa kanyang kritika sa mga panahon ng kanyang pag-aaral. Ang makauring usapin tulad ng paggawa, neoliberalismo, organisadong pagkilos, rebolusyon, sosyalismo, komunismo, at iba pa ay paratihang nasa hayagang pagkalatag ng kritika ni San Juan. Ito na nawawala sa araling kultural ay hindi nawalay at nawala sa kritika ni San Juan. At nang muli itong natagpuan sa araling kultural, masasabing ang trajektori ng kritika ni San Juan ay hindi naman naging hindi makabuluhan lalo na sa panahong binibigwas ng anti-Kaliwa, anti-uri, anti-marxismo ang mga aplikasyon ng kritika dahil ito rin ang ginagawa ng aktwal na may kapangyarihan sa politika, ekonomiya at kultura.

Ang kondisyon ng posibilidad na tinutukoy ni San Juan ay nakaugnay din sa limitasyon at posibilidad ng kritika, na maaring ang elitista o popular na kultura ay magkaroon ng batayan para sa sabayang kooptasyon at subersyon. Ito rin ay nagsasaad ng dialektikang ugnay ng elitista at kulturang popular, hindi bilang magkatunggaling entidad kundi sabayang nakakaimpluensya at naiimpluwensyahan ng kapatirang entidad. Dahil kinikilala ni San Juan ang materialidad ng kosmos ng makauring pagsusuri-na may lahok at substansasyon ng paggawa, pribadong pagaari, ekonomiyang politikal, at iba pa-matutunghayan ang aktwal na hierarkiya, 
segrerasyon, kooptasyon, at posibilidad sa radikalisasyon ng dalawang entidad. Ang makauring salik at kabuuang pagsusuri ang siyang nagtatakda ng posibilidad at imposibilidad ng pangkalahatang kooptasyon at subersyon. At kung ganito, matibay na nakaugnay ang anumang pagsusuri sa elitist at popular na kultura batay sa materialidad ng mga kondisyon ng posibilidad o kung paano ang material na kondisyon ang siya ring kondisyon ng pag-aalsa't pagkakahon ng dalawang ito.

Ang propensidad ng kulturang popular ay matutukoy sa ilang spesipikong larangan at kalakaran: urban na antropolohiya, pangangailangan ng etnograpiya't field work, nagtatala ng posibilidad at imposibilidad (ang "kondisyon ng posibilidad" para kay San Juan) ng negosiasyon at subersyon, at kung ganoon, ang tibay at tamlay ng ugnay nito sa konsumerismo at ang kaakibat na mas malaking usapin ng kalakarang kapitalismo, na titimo sa soft-sell ng gitnang uring aspirasyon at sa mas mayorya, mas mababang kalidad at akses sa mga tsanel ng pagkamamamayan. Kung gayon, ang usapin ng kulturang popular na umunlad at nakatindig sa panahon ng pagkasulat ng sanaysay na ito ay nakapanghahamig din ng usapin ng mabuting pamamahala (good governance) bilang isa pa ring pag-aangkla ng estado sa pagbapor, pagpapalaganap, at pagbibigay ng boto ng kumpyansa sa kapitalismo. Hindi hiwalay ang sinisiwalat ng mga pambansa at lokal na administrasyon sa kanilang retorika ng daang matuwid, walang korapt, laban sa korapsyon, urban renewal, cultural heritage, automated polls, clean at honest na eleksyon, e-governance, usaping pangkalusugan, kalidad ng buhay, akses sa edukasyon at serbisyong publiko, at iba pa ang paglalatag ng kondisyon ng posibilidad sa pagkamamamayan: kung maykaya, merong entitlement dahil meron rin namang ipinapasang pasanin sa pasanin dahil may pangangailangan ng finansyal na kapasidad para sa asersyon ng akses; kung mahirap, pakiusapan, nakabatay sa dole-out na politika at trickle-down na ekonomiks (kung aabot).

At ito ang etikang tinatanganan ng pagsusuri at kritika ng kulturang popular. Sa pagsasalansan ng mga maaring pagsusuri, may titindigan na inaakalang pinakakailangan at pinakaangkop para sa paghamig ng pinakamatalas na pagsusuri. Ito ang valwasyon na mas inaangat ang pagdemanda ng akawntabilidad sa mga entidad ng negosyo, aparato ng estado, at kapitalismo; ang paglulunan sa inaakalang subersibong kalakaran ng mga tumatangkilik ng kulturang popular sa mas malaking kontexto na sino ang higit na nakikinabang, at kung paano nga soft-sell at tanggal ang nosyon ng paggawa sa dating ng pagdanas ng mga ito; at kung paano ang individual at kolektibong subersyon ay mas naikakahon kaysa ipinapalagwa nang lubos ng kuntsabahan ng negosyo at estado. Pinapatagos hanggang sa kalamnan at buto ang kulturang popular sa bawat individual kahit pa, tulad sa pelikula, mass media text o pangmaramihan ang target audience nito; pinapagaan ang pagdukot ng pinaghirapang ipunin na pera sa wallet para sa pagswitch on ng aktwal na pagtangkilik sa produkto, ang kulminasyon ng pagnanasa, 
ang akses pass sa global na kabataang gitnang uri. Tulad kay San Juan, ang lugar ko bilang kritiko ay magbigay-babala sa mga praktikal na gawain sa pamamagitan ng pagbibigay ng mapa ng pagdanas, ng pagmumundo, at kung paano rin minumundo ang nilalang at bansa sa penomenon. Matutunghayan na hindi makatarungan ang kondisyon ng posibilidad sa nilalang, na lampaslampasan ang paglamang at pagmamalabis ng hegemoniya, at kung gayon, tulad ng rekurso ng mga kritiko, maglatag ng kritikal na balangkas para sa panghinaharap: pumusta sa kanyang panunuri sa pangangailangan ng kritikal na pagkamulat sa pagdanas, at sa aktwal na kolektibong paglaya.

Ito ang nalilikhang materialidad ng global na negosyo at formasyong estado: ang ipatanggap, ipatangkilik, paboran at gastusan ang sinasambit na unibersalismo ng pagdanas dito sa kulturang popular. Lahat ay may Facebook, at para ganap ang akses sa Facebook, lahat ay inaasahang may kamera sa telepono, mayroong akses sa Wi-Fi at Internet, mayroon bagong literasi na makakapanghamig ng pinakamaraming likes at reposts - pawang sentral para sa kinetisismo ng pagbibigay-laman sa social media platforms, na naisasalin sa miniminang informasyong ibebenta sa iba pang negosyo para higit na makapagbenta ng mga produkto at serbisyo.

\section{SI SAN JUAN AT ANG REBOLUSYONG KULTURAL}

Tila rebolusyong teknolohikal ang nangyari sa praymordial na gamit sa social networks bilang expresyon ng individualidad, at ang pagkaunlad ng gadgets na magpapabilis at paratihang pwedeng magamit ang akses dito. Pero bago pa man humantong ang mundo at karanasan sa virtualisason ng mga ito, nalinaw na ni San Juan ang diin sa rebolusyong kultural bilang integral sa politikal na transformasyon. Ito ang angkla ng kritika ni San Juan, na siya ring maaring paghugutan ng kritika sa kulturang popular. Marxista si San Juan noon at ngayon, hindi naantala ang kanyang marxismo sa pag-agos at paghupa ng marxismo sa pandaigdigan at pambansang antas. Dagdag pa rito, nananalig din ang kritika ni San Juan sa spesifisidad ng Maoismo sa kondisyon ng posibilidad ng bansa, ang malakolonyal at malapyudal na katangian nito dulot ng tatlong saligang suliranin na patuloy na kinakaharap nito-ang imperialism, burukrata kapitalismo, at pyudalismo. Kung gayon, ang usapin ng uri ay tumatagos sa kritika, at humahantong sa usaping ang kritika ay kasangkapan sa rebolusyonaryong gawain, at katuwang sa pag-aakda ng isang sosyalistang pambansang bukas. Kabahagi ang kritika ni San Juan sa artikulasyon ng pambansang demokrasya o ang layon ng kilusang mamamayan ng atwalisasyon ng pagdanas ng demokrasya sa pambansang antas. 
Ang rebolusyong kultural, kung gayon, ay isang internal at external na adhikain ng nakikisangkot na marxistang intelektwal, tulad ni San Juan. Internal dahil kinakailangan ng pagbabagong-isip at gawi sa mga nakapaloob sa kilusang mamamayan at yaong kayang hamigin para sa layunin ng kilusang ito. Ginagawang pambala ni San Juan ang kanyang mga akda para magkaroon ng lehitimong pagdanas sa marxistang kritika, at ang pagsipi sa kanyang akda bilang dugtong na kumbersasyon sa kondisyon ng posibilidad nito sa kritika. External dahil kinakailangan din ng rebolusyong kultural para sa internasyonalismong proletaryado na siyang layunin ng global na marxistang kilusan. Ito ang lohikal na tunguhin ng pagpupursigi sa lokal, pambansa, at sa kaso ni San Juan, pati sa internasyonal na antas-ang internasyonal na pagtanggap sa kondisyon ng posibilidad sa sosyalismo bilang "another world out there," bilang sabayang inside out (nakalabas na loob) na pahiwatig na ang loob ay kelangang may kaugnayan sa mas malaking nakalabas, at outside in (nakapaloob na labas) para may akses ang labas sa dinamismo ng loob.

Ang rebolusyong kultural ay transformasyong panlipunan at historikal. Binabago nito ang nakagawiang panlipunang relasyon na hindi pantay, hindi makatarungan, at disadbentahe sa mayorya tungo sa isang mas kolektibong egalitaryong panlipunang kaayusan. Historikal dahil transitioning nga itong kaayusan mula sa kasalukuyang bulok at mapagbukod na lipunan sa ilalim ng pyudal at kolonyal na pambansang ayos, tungo sa isang sosyalistang formasyon at transformasyon. Sa China nagmula ang karanasan sa rebolusyong kultural nang bumagsak ang Great Leap Forward bilang rektifikasyon sa rebisyonismong namamayani nitong mga panahon. Para kay Mao, ang rebolusyong kultural ay paratihan ding panunumbalik sa prinsipyo ng marxismo. Ang rebolusyong kultural ni San Juan ay nakabatay kay Mao at sa sulatin ni Amado Guerrero sa Philippine Society and Revolution na siyang naglalatag ng batayang prinsipyo at aralin para sa mga aktibista ng pambansang demokrasya.

Ang rebolusyong kultural ay "permanenteng rebolusyong kultural" na may sakop sa pagtataguyod ng pambansang demokrasya, sa paglalatag ng sosyalistang kondisyon, at tungo sa komunismo. Nag-iiba ang partikularidad pero may katangiang unibersal sa bawat yugto ng rebolusyonaryong gawain ang rebolusyong kultural. Kung magpakaganito ang katangian ng rebolusyong kultural, lalong pinapatalas ni San Juan ang layon tungo sa politikal na araling kultural at politikal na kulturang popular. Ang una ay ang kapasidad ng kritikal na balangkas na makapag-ambag sa pagsusuri ng politika ng kultura, kultura ng politika tungo sa malawakang pagbabagong historikal at panlipunan. Ang ikalawa ay ang formasyon ng politikal na kulturang popular sa hanay ng pambansang demokratikong kilusan na maaring nanghihiram o nanghahamig sa transformasyon ng kulturang popular sa kasalukuyang antas at kalidad nito. Malinaw din na para sa rebolusyong kultural 
ay kinakailangan ng mga kadre para sa rebolusyonaryong gawain, kasama ang mga nakikisangkot na intelektwal at kritiko rito.

Ang pambansang demokratikong pagsusuri, tulad nang naisagawa at patuloy na ginagawa ni San Juan, ay hango sa linya ni Mao na "mula sa masa tungo sa masa" na may pakahulugang ang kalakaran ng hanay mula sa ibaba ay itataas ang kritikalidad sa pamamagitan ng pag-aangkop sa mga nabanggit na teorya't konsepto at rebolusyonaryong diin, at muling ibabalik o makakabalik ang pagsusuri sa mismong pinaghahalawan ng texto at objek ng karanasan. Ito ang dialektikal na kilos ng kritika sa kulturang popular na sinasambit ng kritika ni San Juan.

Sa kabilang banda, ang rebolusyonaryong gawain ay may pakahulugan din ng pagpapalawig at pagpapalaganap ng mismong pambansang demokratikong linya at pagsusuri tungo sa isang popular na odyens. Ibig sabihin, lahat ng pwedeng mahamig sa pagsusuri-at ang akto ng kritika at pagbabasa ay akto rin ng pag-eensayo sa rebolusyon at rebolusyonaryong gawain-ay dapat aktibong maipaloob at mapabilang para sa kolektibong pagbabago. Nangangahulugan ito ng makabagong artikulasyon sa mga metapora at realidad ng rebolusyon, pambansang demokrasya, sosyalismo, kilusang mamamayan, armadong pakikibaka, sosyalismo, at komunismo bilang kasangkapan sa paglikha ng ibang posibilidad mula sa mapanupil na kasalukuyan. Ito ang makabagong layon ng rebolusyonaryong gawain sa rebolusyong kultural: ang aktwal at figuratibong ibenta, paunlarin, pagyamanin, paghalawan ang karanasan sa rebolusyon. Sa madaling salita, ang kritika ng kasalukuyan at hegoniya ng naghaharing kaayusan ay dapat maisalin sa kritika ng pambansang demokratikong pagbabago.

\section{QUO VADIS, SAN JUAN AT KULTURANG POPULAR}

Malinaw kay Mao at kay San Juan na ang lahat ng gawain at kaisipan ay pagpanig sa makauring ideolohiya. Ang mga reaksyonaryong pagsusuri, halimbawa, sa formalismo't bagong kritisismo, maging sa postmodernismong walang makauring pihit, ay partisano sa naghaharing hegemoniya. At kung gayon, walang kahihiyan na ang pagsulat sa pambansang demokratikong kritika ng araling kultural ay partisano rin, at etikal ang pagiging partisano nito: para sa mayoryang anakpawis, katutubo, peti burgesya at pambansang burgesya kayang mahamig para sa pambansang pagbabago tungong sosyalismo.

Bakit kamo? Paliwanag ni San Juan sa Towards a People's Literature, "In the Philippines today, art and literature are matters of life and death, serving either the liberation of the vast majority or the continued domination by the privileged elite. 
Which side shall we chose?" Usaping buhay at kamatayan ang maging taumbayan sa bansa, usaping ipinagkakait na luho ang pagiging mamamayan dito, usaping mitikal ang pinapaniwalang formasyon tungo sa higit na pagbibigay-pribilehiyo sa individual at paglusaw sa kolektibo, lalo na ang may politikal na kalidad. Sa pang-araw-araw na usapin, sa pagpapalit ng faksyon ng naghaharing uri bilang pambansang administrasyon, sa patuloy na pag-atake sa politikal na posibilidad at kapasidad ng kilusang mamamayan, kasama ang armadong kilusan, malinaw ang isinasaad na etika ng pagpanig ni San Juan, na kung kritiko ka, sa bawat kritisismong likha, sa kabuuang kritika, ito ay pagpanig laban sa karahasan ng estado. Walang takot na isinasaad ni San Juan ang kanyang pagpanig sa mga antas ng pakikisangkot sa pambansang demokrasya, pati sa napakahalagang armadong sangkap nito. Kung pinapatay na sa pang-araw-araw ang mga taumbayan sa "mabuting pamamalakad" ng pambansang administrasyon at kapitalismo, at sistematikong pinapaslang ang mga politikal na figura nito para pahinain ang kilusan, ano pa ang papel ng pagdududa, bakit hindi pa papanig sa politika ng buhay at banyuhay ng pambansang demokratikong kilusan kaysa sa nekropilya ng estado?

At ganito rin ang mga kondisyon ng posibilidad na isinasaad sa kritika ngkulturang popular. Sangay rin ng nekropilya nito ang estado, o franchise ng funeraria na binibigyan-pribilehiyo ang diskurso ng kamatayan sa pamamahala ng taumbayan. Mahalaga ang kritika ni San Juan sa diskursong kulturang popular kahit nga ito hindi direktang nagsulat sa kulturang popular. Nagtagpo ang sinimulan at pinaunlad niyang punto sa kung saan humantong ang diskursong kulturang popular pagkatapos itong magpaligoyligoy sa iba't ibang yugto ng araling kultural. Politikal ang pagsusuri ni San Juan batay sa pagsusuri ng politika, na nangangahulugan ng pangangailangan ng transformasyon tungo sa may katarungang panlipunan. Isinaad din niya ang pangangailangan ng imahinaryong historal na transformasyon, na hindi hiwalay ang kritika sa pag-akda ng makatarungang pambansang kinabukasan. Si San Juan na konsistent sa kanyang politika ay paratihan sa kanyang kritisismo at kabuuang kritika reiteratibo sa politikal na gawain at panawagan. Matatagurian si San Juan na "angel ng kasaysayan" (angel of history ni Walter Benjamin) na nagpapabagsak sa lupa sa sinumang inaakalang umuunlad at nakalutang na ang pambansang ekonomiya, na ang nahalal na politiko ay tunay na makakapagpabago ng daandaang taong sistema ng bansa, na ang pantawid-gutom na mga programa ang solusyon sa poverty alleviation, na ang infrastruktura at diversidad ng tao at restaurant sa Bonifacio Global City ay pagpakilala na ang bansa ay First World na, ang pagpaslang sa mga Lumad ay kinakailangan para higit na madusta ang likasyamang nasa kanilang poder, na ang paglabag sa karapatang tao ng ordinaryong mamamayan at politikal na pagpaslang ng mga aktibista at peryodista ay kolateral na pinsala sa pagtataguyod ng kapayapaan, na ang malawakang trafiko sa Metro Manila ay sinyales na umuunlad na nga ang bansa, na ang pagiging number 1 sa Facebook ng mga Filipino sa dami ng oras na inilalaan dito ay hudyat ng 
pamamayagpag ng kabataang techies, na ang 15 milyong OCWs o overseas contract workers ay nahihiwalay sa kanilang pamilya sa ngalan ng pag-aambag sa pambansang ekonomiya't pagkahirang bilang bagong bayani, na ang sunshine industry na call center at ang profesyon na call center agent ay hindi pagsadlak sa milyonmilyong kabataan sa isang walang pag-unlad at nakapresong kinabukasan, at iba pang ginagawa ng estado sa ngalan ng kaunlaran at kapayapaan, at sa higit na ngalan ng negosyo, kita at kapitalismo.

Ang personal na sanaysay na ito ay isang paglalakbay, kasama ng multo. Ngayon na lamang nagretiro si San Juan, sa mas mahaba't personal na pagbabalik sa Pilipinas, nagkaroon ng pagkakataon na magkaroon ng relasyon higit pa sa loob ng kritika. At ito ang ikinatutuwa ko sa paglalakbay simula sa huling bahagi ng dekada I980, na sa pagbabaliktanaw tulad nito, may mga naging panandangbato na gumiya sa sariling paglalakbay kundi man paglalayag sa kritika at pagiging mamamayan sa bansa, pagiging nilalang sa bayan, at patuloy na gumigiya sa aking politikal na edukasyon, o edukasyon sa higit pang politikal na gawain bilang kritiko, manunulat, mamamayan, at nilalang para sa sambayanan. At kung bakit ito ang paninindigan ko kung bakit ako nagsusulat, at piniling magsulat hinggil sa kulturang popular at ang komplexidad ng gawaing ito (kailangan ito sa mga bago at pabago-bagong tinatangkilik at paraan ng pagtangkilik). Nagpapasalamat ako sa mga multo na ang kanilang presensya ay nagpapaalaala sa akin ng mga ito.

Sa kritika ni San Juan, tulad ng panawagan ng mamamayan sa sariling hanay, kaisa na rin ako sa pagtindig, "Makibaka, wag matakot!" 